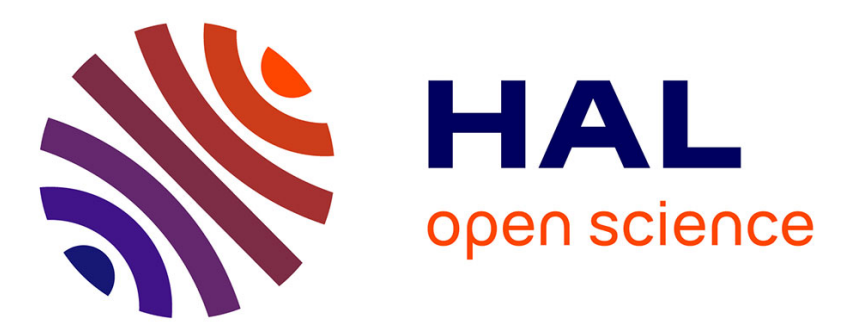

\title{
Two-wave mixing in an erbium-doped fiber amplifier for modulation depth enhancement of optically carried microwave signals
}

Stéphanie Norcia-Molin, Sylvie Tonda-Goldstein, Daniel Dolfi, Jean-Pierre Huignard, Robert Frey

\section{To cite this version:}

Stéphanie Norcia-Molin, Sylvie Tonda-Goldstein, Daniel Dolfi, Jean-Pierre Huignard, Robert Frey. Two-wave mixing in an erbium-doped fiber amplifier for modulation depth enhancement of optically carried microwave signals. Optics Letters, 2006, 31 (3), pp.299-301. hal-00867099

\section{HAL Id: hal-00867099 \\ https://hal-iogs.archives-ouvertes.fr/hal-00867099}

Submitted on 27 Sep 2013

HAL is a multi-disciplinary open access archive for the deposit and dissemination of scientific research documents, whether they are published or not. The documents may come from teaching and research institutions in France or abroad, or from public or private research centers.
L'archive ouverte pluridisciplinaire HAL, est destinée au dépôt et à la diffusion de documents scientifiques de niveau recherche, publiés ou non, émanant des établissements d'enseignement et de recherche français ou étrangers, des laboratoires publics ou privés. 


\title{
Two-wave mixing in an erbium-doped fiber amplifier for modulation depth enhancement of optically carried microwave signals
}

\author{
S. Norcia-Molin, S. Tonda-Goldstein, D. Dolfi, and J.-P. Huignard \\ Thales Research \& Technology, Domaine de Corbeville, 91404 Orsay Cedex, France \\ R. Frey \\ Laboratoire Charles Fabry de l'Institut d'Optique, Centre Scientifique Paris-Sud, Bâtiment 503, \\ 91403 Orsay Cedex, France
}

Received June 20, 2005; accepted September 13, 2005

\begin{abstract}
We experimentally and theoretically analyze an original method based on two-wave mixing in an erbiumdoped fiber amplifier for optical carrier reduction of microwave signals. $75 \%$ optical carrier attenuation has been observed, and a $10 \mathrm{~dB}$ modulation depth increase of the microwave signal is experimentally demonstrated. Moreover, calculated results are in good agreement with measurements and predict that up to $80 \%$ carrier attenuation is easily possible. (C) 2006 Optical Society of America

OCIS codes: $190.7070,060.4080$.
\end{abstract}

Optoelectronic links to be implemented in the new generation of radar and radio-over-fiber systems have to be highly linear over a large dynamic range. Indeed, because of the limited linearity of conventional integrated optics Mach-Zehnder modulators (MZMs), the optical carrier has to be weakly modulated at microwave frequency $f_{m}$ in order to avoid any spurious signals at harmonic frequencies $2 f_{m}$, $3 f_{m}, \ldots$. The transmitted signal is then composed of a low modulated part and a large dc component. This last component is mainly responsible for the signal distortions occurring in high-speed and lowsaturation-level photodetectors. Thus highperformance optoelectronic links in future RF systems require selective optical carrier reduction of weakly modulated microwave signals. Dynamic carrier filtering was demonstrated by use of stimulated Brillouin scattering ${ }^{1,2}$ as a nonlinear effect in optical fibers, permitting more than $40 \mathrm{~dB}$ modulation depth enhancement of optically carried microwave signals. The limitations of such an approach are linked to the facts (i) that the stimulated Brillouin scattering nonlinearity has to be pumped by a signal modulated in the $\mathrm{RF}$ domain, requiring a quite high power level at the input of the MZM, and (ii) that reducing the carrier also results in a net reduction of the final detected RF signal, requiring additional optical amplification.

According to these considerations, we present in this Letter a solution for modulation depth enhancement of optically carried microwave signals by using two-wave mixing and beam coupling phenomena in an erbium-doped fiber amplifier, which at the same time combines carrier reduction and optical amplification. Nonlinear two-wave mixing in bulk amplifiers was analyzed and demonstrated in Refs. 3-7 for dynamic holography and phase-conjugate laser cavities. Two-wave mixing by gain saturation in fiber amplifiers has also been investigated, ${ }^{8-11}$ including demonstration of a narrow-bandpass filtering function in
Ref. 12. Nevertheless, no results concerning a modulation depth increase of optically carried microwave signals have yet been reported.

The all-fiber experimental setup implemented to demonstrate modulation depth enhancement of optically carried microwave signals by two-wave mixing in an erbium-doped fiber amplifier is described in Fig. 1. A polarization-maintaining erbium-doped fiber $\left(\mathrm{Er}^{3+} \mathrm{PMF}\right)$ of length $L=5 \mathrm{~m}$ is optically pumped at $980 \mathrm{~nm}$. Gain into the $\mathrm{Er}^{3+} \mathrm{PMF}$ is obtained by optical pumping at $980 \mathrm{~nm}$. A single-frequency laser source at $1.5 \mu \mathrm{m}$ is divided into two beams by an optical coupler. The most intense beam (the pump) passes an optical circulator (OC) from port 1 to port 2 before injection into the $\mathrm{Er}^{3+} \mathrm{PMF}$ at $z=0$ (the $z$ axis is the light-propagating axis in the fiber). The weaker beam (the probe signal) is used as a microwave signal optical carrier: it passes a MZM for intensity modulation at microwave frequency $f_{m}$. This modulated

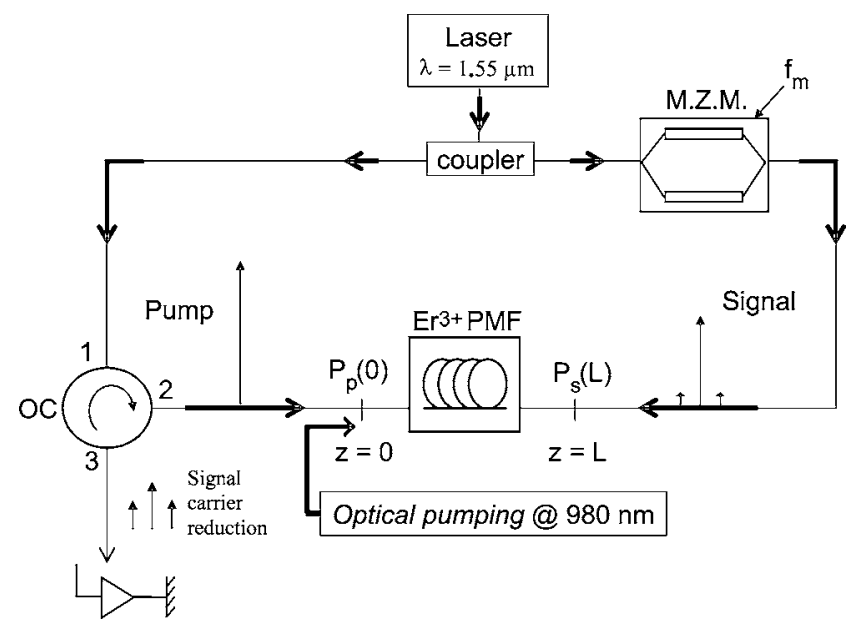

Fig. 1. Experimental setup. $\mathrm{Er}^{3+} \mathrm{PMF}$ numerical aperture $\mathrm{ON}=0.16 \pm 10 \%$, core diameter $\varnothing=4 \pm 1 \mu \mathrm{m}$, cutoff wavelength $\lambda_{c}=900 \pm 100 \mathrm{~nm}$, losses $7 \mathrm{~dB} / \mathrm{m}$ at $980 \mathrm{~nm}$ and $10 \mathrm{~dB} / \mathrm{m}$ at $1531 \mathrm{~nm}$. 
signal is injected into the $\mathrm{Er}^{3+} \mathrm{PMF}$ at $z=L$, travels in from $z=L$ to $z=0$, and finally passes the OC from port 2 to port 3. Since these two coherent optical waves (pump and probe signals of the same wavelength $\lambda \sim 1.55 \mu \mathrm{m}$, the same polarization stateaccurately controlled on each path-and of coherence length $L_{\text {coh }}>L$ ) are injected in reverse directions at both ends of the $\mathrm{Er}^{3+} \mathrm{PMF}$, they give rise to optical interferences in the fiber. This leads to a spatial periodic modulation of the gain all along the fiber, by gain saturation. This dynamic photoinduced gain grating causes self-diffraction and coupling of the counterpropagating waves. In particular, the diffraction of the intense pump beam creates a backpropagating wave in the direction of the weak probe beam. In the gain medium this backpropagating selfdiffracted wave is $\pi$ shifted with respect to the probe wave. ${ }^{13}$ Consequently, because of destructive interferences, the detected signal intensity at port 3 of the OC (Fig. 1) is decreased. In this way the photoinduced dynamic gain grating and the related beam coupling allow selective reduction of the optical carrier of the probe beam. Let us note that the modulation sideband interferes with the optical carrier, giving rise to a very fast moving grating that is not recorded as a spatial gain modulation in the Erdoped fiber owing to the population grating lifetime constant of $12 \mathrm{~ms}$. Futhermore, in our experiment the typical length of the doped fiber is about $5 \mathrm{~m}$, which results in a frequency selectivity of the gain grating of about $30 \mathrm{MHz}$. The principle of modulation depth enhancement by two-wave mixing in an amplifier medium is illustrated in Fig. 2. $A_{s}(z, v)$ and $A_{s}\left(z, v \pm 2 \pi f_{m}\right)$ stand, respectively, for the amplitudes of the optical carrier and of the modulation sidebands. While propagating through the amplifier (from $z=L$ to $z=0$ ), the sidebands receive their gain mean value $G^{1 / 2}$ (which may avoid the use of an optical postamplification module). Because of filtering by the gain grating, the optical carrier amplitude, although amplified by $G^{1 / 2}$, is affected by a reduction coefficient $\left(1-\eta^{1 / 2}\right)$ ( $\eta$ being schematically the gain grating diffraction efficiency in the nondepleted pump regime), leading to a modulation depth increase of the signal. The use of optical fiber amplifiers allows recording of long (and consequently highselectivity) gain gratings.

An analysis of the gain grating including the interaction of the propagating pump and $\mathrm{RF}$ modulated probe waves will be presented. Coupled-wave differential equations (1) and (2) for the amplitudes of two counterpropagating interfering waves affected by

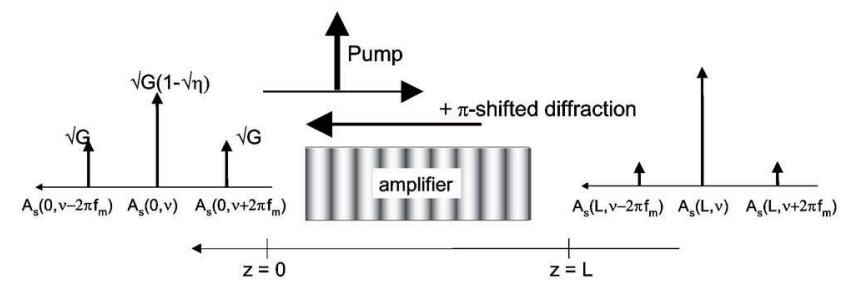

Fig. 2. Principle of optical modulation depth enhancement by two-wave mixing in an amplifier medium. gain saturation in the fiber amplifier have been deduced from previous work ${ }^{9}$ :

$$
\begin{gathered}
\frac{\mathrm{d} A_{p}}{\mathrm{~d} z}=\gamma_{0} A_{p}-\gamma_{1} A_{s}, \\
\frac{\mathrm{d} A_{s}}{\mathrm{~d} z}=-\gamma_{0} A_{s}+\gamma_{1} A_{p} .
\end{gathered}
$$

$A_{p}$ and $A_{s}$ stand, respectively, for pump and probe signal wave amplitudes; $\gamma_{0}$ and $\gamma_{1}$ are, respectively, the nonlinear gain coefficient and the nonlinear coupling coefficient. Coefficients $\gamma_{n}$ are given by $\gamma_{n}$ $=\left(g_{0} / 2\right)(1 / D)\left[\left(1+I_{\Sigma}-D\right) / I_{M}\right]^{n}$ (with $\left.n=0,1\right)$, where $g_{0}$ is the amplifier small-signal gain, $D=\left[\left(1+I_{\Sigma}\right)^{2}\right.$ $\left.-I_{M}^{2}\right]^{1 / 2}, \quad I_{\Sigma}(z)=\left[\left|A_{p}(z)\right|^{2}+\left|A_{s}(z)\right|^{2}\right] / I_{\text {sat }}, \quad$ and $I_{M}(z)$ $=2\left(\left|A_{p}(z) A_{s}(z)\right| / I_{\text {sat }}\right)\left(I_{\text {sat }}\right.$ being the amplifier saturation power density). These equations have been solved numerically with a fourth-order Runge-Kutta algorithm. The calculation has been made under the assumptions that (i) the gain profile has a Lorenztian shape, (ii) optical frequencies are close to the resonance frequency of the gain medium so that no index grating contribution is considered, and (iii) incident optical pump power is small with respect to the saturation power density of the gain medium $\left(\left|A_{p}(z)\right|^{2}\right.$ $\left.\ll I_{\text {sat }}\right)$. Under such conditions pump and signal wave interactions have been studied as functions of the gain $g_{0} L$ of the medium and also as functions of the optical powers of the components that coherently interfere. Also, the presented model is based on spatial gain modulation. But, as analyzed in Ref. 14, the index contribution in an efficiently pumped fiber amplifier can also contribute to two-wave mixing beam interaction.

Results are presented in the following part and are compared with measurements. The probe signal mean output power $\left[P_{s}(z=0, \nu)\right]$ is given with and without the existence of a gain grating in the fiber, as a function of the injected pump power $\left[P_{p}(z=0, \nu)\right]$ normalized to amplifier power saturation $P_{\text {sat }}$. Absence of the gain grating is obtained experimentally when the pump and the signal beams are mutually incoherent. Amplifier saturation power $P_{\text {sat }}$ has been determined experimentally. Results are presented in Fig. 3 for $g_{0} L=5.4\left(P_{\text {sat }} \sim 1.5 \mathrm{~mW}\right)$ and $P_{p}(0) / P_{s}(L)$ $=200$. In both cases (with and without the gain grating) probe powers $P_{s}(0)$ are increasing functions of the injected pump power. For a given value of $P_{p}(0, \nu)$ the transmitted signal power decreases in the presence of the gain grating, which demonstrates the relative attenuation of the signal beam. Amplified Rayleigh scattering into the $\mathrm{Er}^{3+} \mathrm{PMF}$ may explain the small discrepancy between experiments and theory.

Calculated ratios of $P_{s}(0)$ with and without the gain grating correspond to the relative optical carrier attenuation and are reported in Fig. 4 for various values of $g_{0} L$. The calculation is also compared with the measurement for $g_{0} L=5.4$. Satisfactory agreement between model and experiment is obtained for ratios $P_{p}(0, \nu) / P_{\text {sat }}$ in the range of $10^{-1}-10^{-4}$. For lower val- 
ues of the ratio $P_{p}(0, \nu) / P_{\text {sat }}$, the measurement accuracy is too low to permit correct comparison with theory. Let us note that the phase shift due to thermal gradients and vibration of the fiber amplifier may create perturbations that affect the stability of the beam coupling of the interfering contrapropagating waves. From Fig. 4, the larger the gain $g_{0} L$, the better the signal attenuation. A significant signal attenuation corresponds to $80 \%$ of the maximum reachable attenuation. With this criteria and for $g_{0} L=5.4$, the ratio $P_{p}(0, \nu) / P_{\text {sat }}$ is in the range of $10^{-4}-10^{-1}$, which permits $70 \%$ relative attenuation $(5.2 \mathrm{~dB})$. For $g_{0} L=10$, the required ratio $P_{p}(0, \nu) / P_{\text {sat }}$ is in the range of $10^{-5}-10^{-1}$.

Measurements of the modulation depth increase have been analyzed as a function of the ratio between injected optical powers $P_{p}(0, \nu) / P_{s}(L, \nu)$, for $g_{0} L=4$, and $P_{s}(L, \nu)=30 \mu \mathrm{W}$. Results are reported in Fig. 5 . The transmitted modulated signal intensity is detected by a photodiode at port 3 of OC. The input optical signal modulation depth at $z=L$ is $m_{\text {in }}=0.5 \%$, corresponding to a ratio $P_{s}(L, \nu) / P_{s}\left(L, \nu+2 \pi f_{m}\right)$ $\sim 0.25 \%$. The maximum output optical signal modulation depth reached at $z=0$ is $m_{\text {out }}=4.6 \%$, corresponding to a gain on the modulation depth close to $10 \mathrm{~dB}$, with an overall increase in RF signal power of

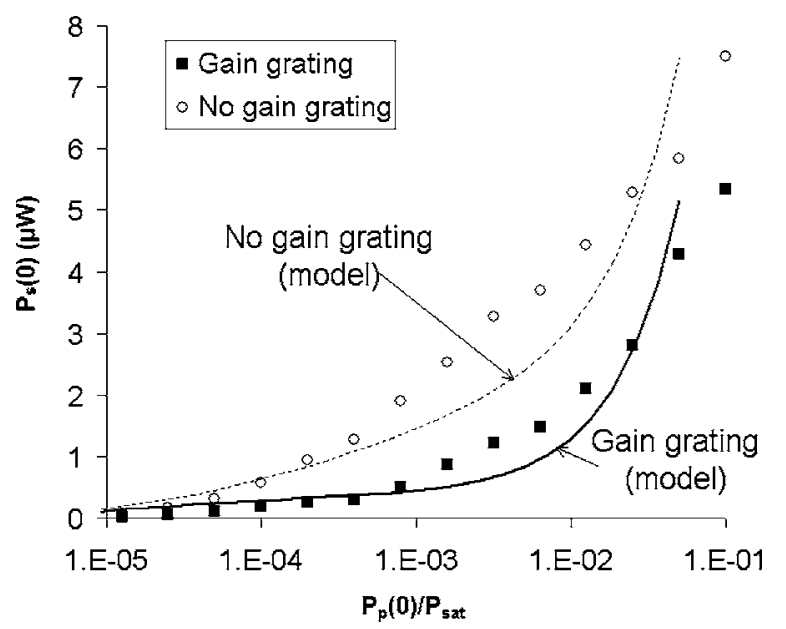

Fig. 3. Measured and calculated output probe power, with and without the existence of a gain grating, as a function of the injected pump power $\left(g_{0} L=5.4\right)$. The probe is not modulated.

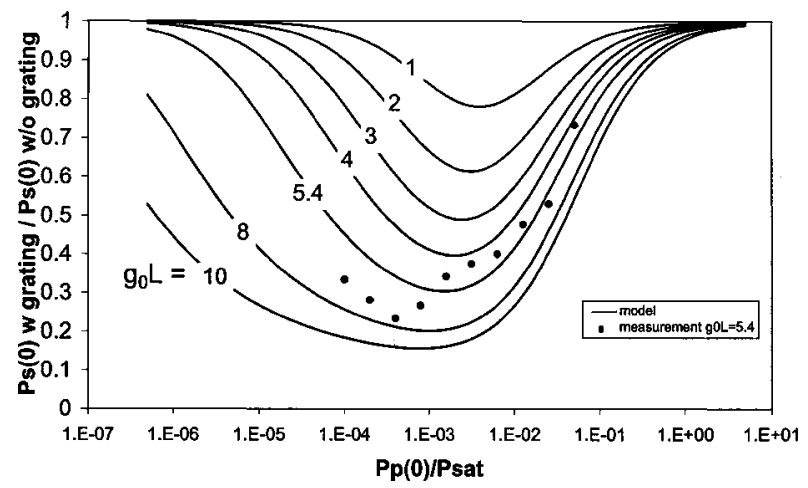

Fig. 4. Relative optical carrier attenuation, as a function of injected pump power, and for various values of fiber amplifier gain $g_{0} L$.

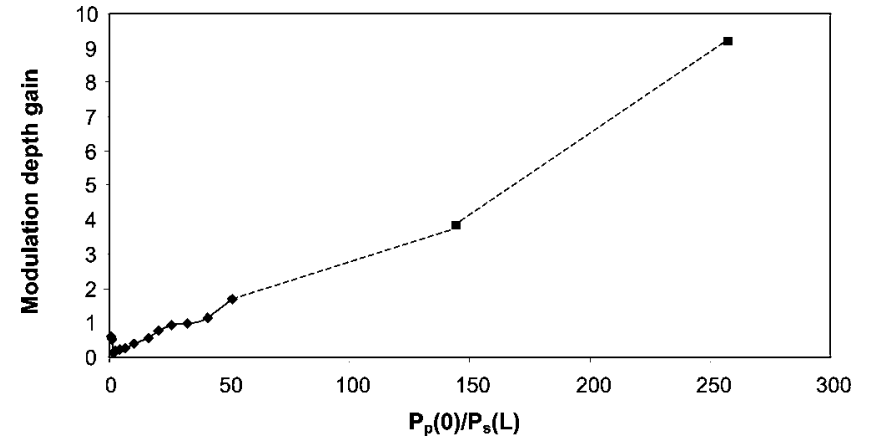

Fig. 5. Measured modulation depth gain as a function of the ratio of injected pump and probe powers for modulation frequency $f_{m}=7 \mathrm{GHz}$ of the probe and $g_{0} L=4$. The two points on the dotted lines stand for measurements not fulfilled by the presented model.

nearly $20 \mathrm{~dB}$. In Fig. 5 the condition of no gain saturation $\left(I \ll I_{\text {sat }}\right)$ considered in the model is fulfilled only for the power ratio range $P_{p}(0) / P_{s}(L)<50$.

In conclusion, we have experimentally and theoretically analyzed a two-wave mixing interaction between $\mathrm{cw}$ and RF-modulated signal beams in a $5 \mathrm{~m}$ long erbium-doped fiber amplifier. This interaction is an original approach for optical carrier reduction of microwave signals. Calculated results are in good agreement with measurements. Adaptive and selective optical carrier attenuation of $75 \%$ has been measured, resulting from beam coupling effects in the fiber amplifier. A $10 \mathrm{~dB}$ increase of the modulation depth of the optically carried microwave signal has been experimentally demonstrated, together with a $20 \mathrm{~dB}$ increase in the RF signal power. The model predicts even higher optical carrier attenuations with improvements of the experimental setup and longer fiber amplifiers for reaching a 100\% modulation depth.

\section{References}

1. K. J. Williams and R. D. Esman, Electron. Lett. 30, 1965 (1994).

2. S. Norcia, S. Tonda-Goldstein, R. Frey, D. Dolfi, and J.-P. Huignard, Opt. Lett. 28, 1888 (2003).

3. A. Tomita, Appl. Phys. Lett. 34, 463 (1979).

4. M. J. Damzen, R. P. M. Green, and K. S. Syed, Opt. Lett. 20, 1704 (1995).

5. A. Minassian, G. J. Crofts, and M. J. Damzen, IEEE J. Quantum Electron. 36, 802 (2000).

6. P. Sillard, A. Brignon, and J.-P. Huignard, IEEE J. Quantum Electron. 34, 465 (1998).

7. P. Yeh, IEEE J. Quantum Electron. 25, 484 (1989).

8. S. T. Fisken, Opt. Lett. 17, 1776 (1992).

9. B. Fischer, J. L. Zyskind, J. W. Sulhoff, and D. J. DiGiovanni, Opt. Lett. 18, 2108 (1993).

10. M. Horowitz, R. Daisy, B. Fischer, and J. L. Zyskind, Opt. Lett. 19, 1406 (1994).

11. M. Janos and S. C. Guy, J. Lightwave Technol. 16, 542 (1998).

12. S. A. Havstad, B. Fischer, A. E. Willner, and M. G. Wickham, Opt. Lett. 24, 1466 (1999).

13. A. Brignon, J.-P. Huignard, Opt. Lett. 18, 1639 (1993).

14. O. L. Antipov, O. N. Eremeykin, A. P. Savikin, V. A. Vorob'ev, D. V. Bredikhin, and M. S. Kuznetsov, IEEE J. Quantum Electron. 39, 910 (2003). 